\section{ALCOHOL, ACETONE AND PROPYLENE OXIDE AS DEHYDRATING AGENTS OR INTERMEDIATE SOLVENTS PRIOR TO EPOXY EMBEDDING OF BIOLOGICAL SPECIMENS \\ Hildegard H. Crowley, University of Denver}

Acetone, alcohol, and propylene oxide have different mechanical and chemical properties. They should not be chosen haphazardly but should be used with care to suit the particular demands of the embedding conditions.

\section{ALCOHOL}

Ethyl alcohol is extremely miscible with water in all proportions. It has a lower viscosity than acetone; therefore it penetrates impervious tissue structures more readily. Epithelium, collagen, and mycobacterium, for instance, are efficiently dehydrated by alcohol. Its use should also be considered for all tissue samples larger than $1 \mathrm{~mm}$ on a side. Alcohol generally solvates lipids more readily and rapidly than acetone. Therefore if lipid structures are of particular interest, the use of alcohol as a dehydrating agent should be avoided if possible.

Alcohol is not indicated as an intermediate agent since it is considered a nonreactive dilutent ${ }^{1}$. Nonreactive dilutents do not become part of the polymerized system. Instead, reagent not driven off by heat will remain in the tissue interfering with polymerization, or in extreme cases cause stickiness. However, sometimes the use of alcohol as an intermediate is demanded by the solubility of the surface of other components of the embedding methods, such as plastic dishes, which will be attacked and dissolved by acetone or propylene oxide. Since degradation of chemical and mechanical properties of the block will ensue due to remnants of alcohol left in the tissue ${ }^{2}$, numerous changes of pure resin are needed to rinse out the alcohol.

\section{ACETONE}

If lipid structures are of importance in the final micro- graph, acetone is generally the dehydrating agent of choice, since in most cases preservation of the specimen will be enhanced. Acetone is a serviceable intermediate agent, as it is more easily deleted from the final embedding mixture than alcohol. However, acetone also interferes with polymerization.

\section{PROPYLENE OXIDE}

Propylene oxide is the most fortuitous intermediate agent for epoxies for numerous reasons. It reduces the viscosity of resin more than alcohol or acetone. This requires its use during infiltration of large or dense tissues. It is, however, thought to be a carcinogen. Its handling requires extra caution as it easily penetrates latex gloves.

Propylene oxide is the simplest of the epoxies. It is a reactive monoepoxy and is a "chain stopper", since it decreases crosslinking density of the epoxy block by shortening the lengths of the diepoxy (Epon) chains ${ }^{3}$. Evaporation from the bloc is limited by the stearic hindrances caused by the crosslinking of epoxies at about $45^{\circ} \mathrm{C}$. Unlike acetone or alcohol, propylene oxide actually becomes part of the polymerized system ${ }^{4}$. Therefore, small quantities left in the block are of no consequence; however, larger amounts will decrease resistance to heat in the electron beam resulting in section instability.

Formulations designed with regard to the WPE numbers of diepoxides and hardeners using propylene oxide, as a monoepoxide dilutant, will polymerize normally as long as the monoepoxide

\title{
Introducing
}
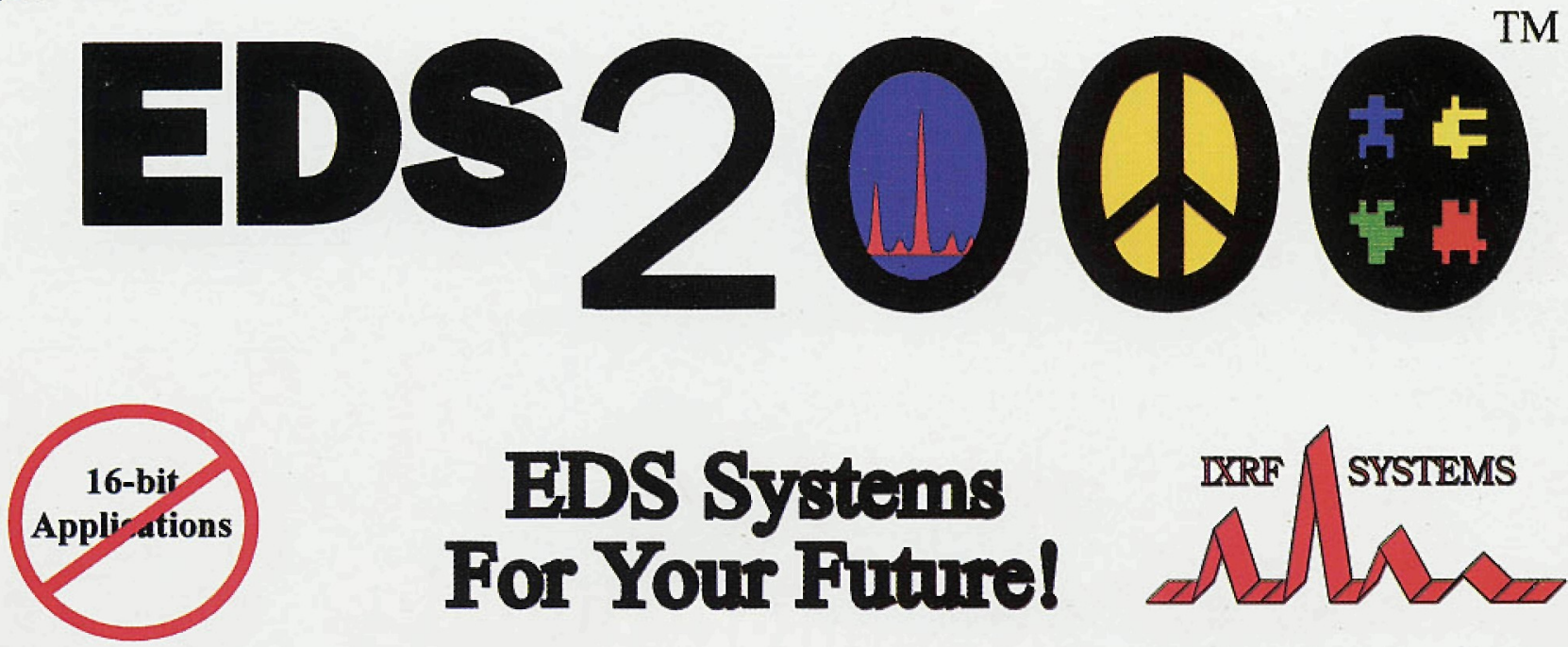

IXRF Systems, Inc. 15715 Brookford Dr. Houston TX 77059 Tel: 281/286 -6485 Fax: 281/286.2660 www.ixrfystems.com EDS2000 and EDX2000 are trademarks of IXRF Systems, Inc. 
is of minimal percentage. As the proportions of monoepoxy increase, the block becomes softer, and more flexible. If the percentage of propylene oxide in the embedding mixture is great enough, the block is so flexible that it can be bent sideways in the chuck of the microtome (unpublished observations).

Inclusion of propylene oxide in the final embedment intensifies the adhesive properties of epoxy formulations. This concept is of crucial importance when embedding sections which adhere to microscope slides ${ }^{5}$. If any propylene oxide is left on the slide when the epon filled capsule is inverted over the section, the tissue will not detach properly with either liquid nitrogen or heat, because the area around the section has become too adhesive and too elastic. Thus understanding the differing chemical and mechanical properties of alcohol, acetone, and propylene oxide will allow the microscopist to make informed, and sometimes, critical, choices correctly.

\section{REFERENCES}

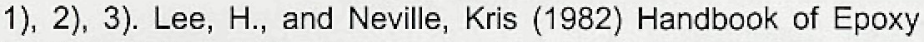
Resins. McGraw Hill, Chapter 13, p13-1 to 13-20.

4) Luft, J. H. (1961), Improvements in epoxy embedding methods, J. biophys. Biochem. Cytol. 9, 409.

5) Crowley, H. H., and Kinnamon, J. C. (1995) Experimental Cell Biology of Taste and Olfaction. Ed. Spielman, A.I., and Brand, J. G. Transmission electron microscopy of gustatory epithelium, $p$ $110-111$

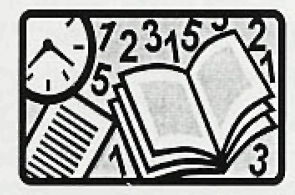

\section{Examining Ferromagnetic Samples In The SEM/EDX \\ Mary Mager, University of British Columbia}

While the presence of any magnetic material in the chamber of the SEM will distort the image to some degree, it is possible to take pictures and do EDX of such samples if a little care is used.

1) Make the sample as small as possible. If it is a powder use only a few grains. If it is a chunk cut it down as small as possible. Stick the samples down well so they can't fly off the stub and stick to the SEM polepiece. I use carbon conductive sticky tabs because they are conductive, so no other treatment of a metal sample is needed, and because they have quite a strong glue. Mounting the sample in epoxy and polishing will also help keep it in place.

2) Keep the working distance as long as possible. If you are doing EDX this distance is fixed, but if you're just imaging, use as long a distance as you can and still get the photo you want. The magnetic interference falls away as the square of the distance from the lens.

3) You will have to correct the astigmatism of the image just before you take the photo. Each time you move the sample the magnetic distortion will change and the resulting astigmatism will change.

4) It is possible to get an EDX analysis from a sample, even if the image is distorted. I once analyzed a super-magnet and got a fine EDX analysis of it, even though the image was just a mad swirl.

With most normal nickel and steel alloys there is really very little problem. We image them all the time.

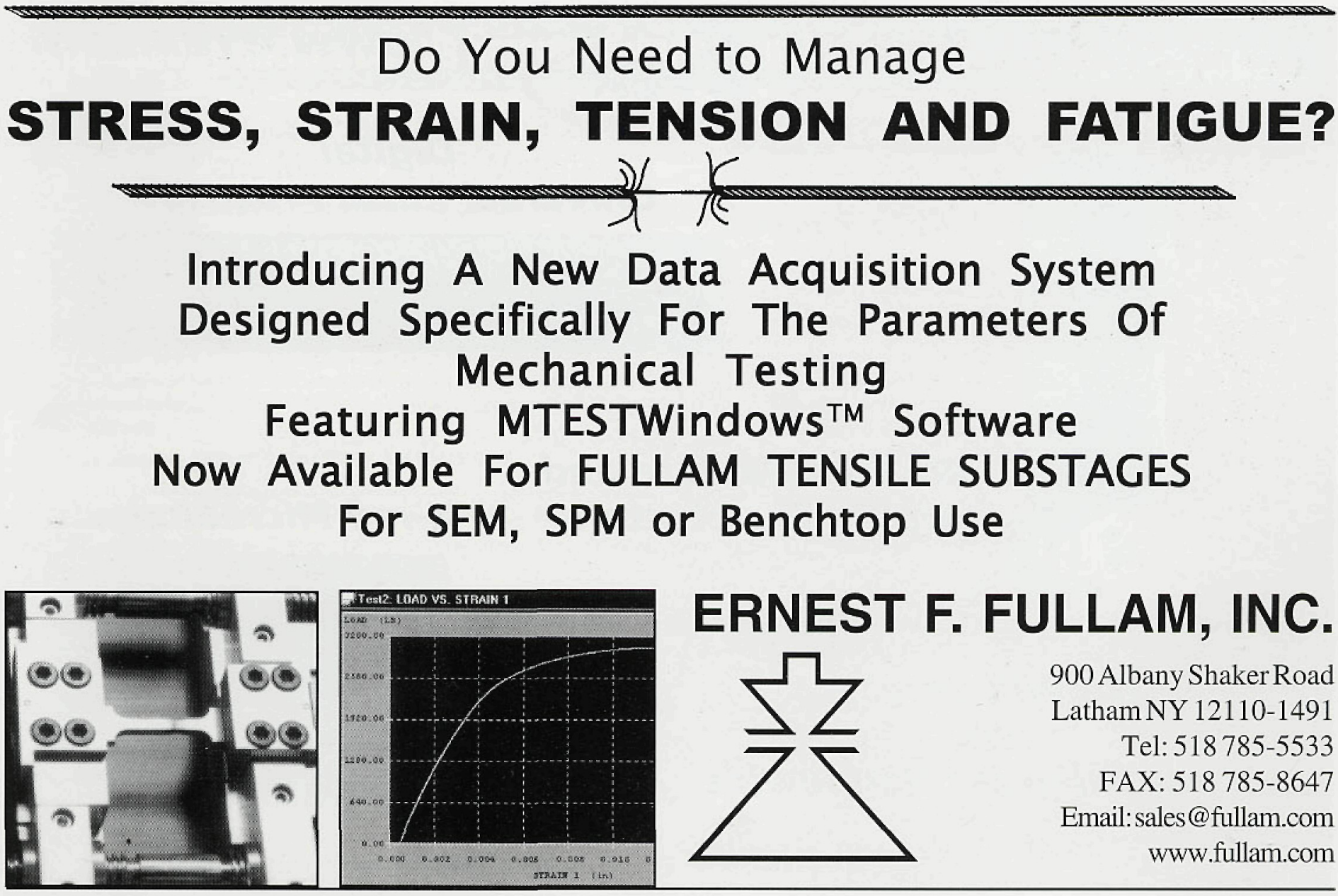

